\title{
Claudin-1 and claudin-2 expression is elevated in inflammatory bowel disease and may contribute to early neoplastic transformation
}

\author{
Christopher R Weber ${ }^{1,3}$, Sam C Nalle ${ }^{1,3}$, Maria Tretiakova ${ }^{1}$, David T Rubin ${ }^{2}$ and Jerrold R Turner ${ }^{1}$
}

\begin{abstract}
Patients with inflammatory bowel disease (IBD) are at increased risk of developing colorectal adenocarcinoma. The factors that result in IBD-associated carcinogenesis are not understood. We hypothesized that altered expression of intestinal epithelial tight junction proteins might contribute to neoplastic progression. Semiquantitative immunohistochemical staining of human biopsies was used to assess expression of the tight junction proteins claudin-1, claudin-2, claudin-4, and occludin in IBD, IBD-associated dysplasia, acute, self-limited colitis (ASLC), and sporadic adenomas. Claudin-1 and claudin-2 expression was elevated in active IBD, adenomas, and IBD-associated dysplasia, but not ASLC. In contrast, claudin-4 expression was elevated in both active IBD and ASLC. Occludin expression was similar to control in all cases. Importantly, in IBD, claudin-1 and claudin-2 expression correlated positively with inflammatory activity. To investigate mechanisms underlying altered claudin expression, $\beta$-catenin activation was assessed as nuclear localization. Like claudin1 and claudin-2, $\beta$-catenin was markedly activated in IBD, sporadic dysplasia, and IBD-associated dysplasia, but was only slightly activated in ASLC. Taken together, these data suggest that $\beta$-catenin transcriptional activity is elevated in chronic injury and that this may contribute to increased claudin-1 and claudin-2 expression. We speculate that increased claudin-1 and claudin-2 expression may be involved at early stages of transformation in IBD-associated neoplasia. Laboratory Investigation (2008) 88, 1110-1120; doi:10.1038/labinvest.2008.78; published online 18 August 2008
\end{abstract}

KEYWORDS: claudin; colon cancer; dysplasia; inflammatory bowel disease; occludin; tight junction

The intestinal epithelial barrier is established by a single layer of cells. These are held together by intercellular junctions that include desmosomes, adherens junctions, and tight junctions. The seal that regulates paracellular permeability is established by the tight junction, which is composed of transmembrane proteins, such as claudins and occludin, and cytosolic proteins, such as ZO-1. When examined by freezefraction electron microscopy, tight junctions are visualized as a network of anastomosing strands within the cell membranes. The number and complexity of these strands is decreased in inflammatory bowel disease (IBD), and this correlates with reductions in barrier function. ${ }^{1}$ The molecular correlates of this reduced barrier function are only beginning to be understood. ${ }^{2-5}$

Although barrier dysfunction in IBD is incompletely understood, ${ }^{2}$ the increased inflammatory cytokine content of mucosa affected by IBD may play a critical role. ${ }^{3,6-9}$ For example, cytokines have been shown to modify the expression and distribution of tight junction proteins. ${ }^{5}$ Both IL-13 and TNF have been reported to increase claudin-2 expression. ${ }^{3,4,8}$ TNF also downregulates expression ${ }^{10}$ and promotes endocytosis of occludin. ${ }^{6,11,12}$ In addition to the effects on barrier function, some data also suggest that these proteins may regulate signaling, gene expression, or other aspects of cellular pathophysiology. ${ }^{13,14}$

Claudin expression is altered in a variety of preneoplastic conditions and frank neoplasms. ${ }^{15-19}$ For example, claudin-4 is elevated in pancreatic adenocarcinoma ${ }^{20}$ and claudin-3 and claudin- 4 are elevated in Barrett's esophagus ${ }^{21}$ and some gastric adenocarcinomas. ${ }^{15}$ As might be expected, claudin protein expression is also correlated with tumor differentiation. ${ }^{22}$ Several studies have evaluated tight junction protein expression in colonic adenocarcinoma, and most have reported increased expression of claudin-1 ${ }^{13,23-25}$ and claudin- $2 .^{24,26,27}$ Claudin-1 expression is a positive prognostic indicator and correlates with lower tumor grade, absence of lymphovascular

\footnotetext{
'Department of Pathology, University of Chicago, Chicago, IL, USA and ${ }^{2}$ Department of Medicine, University of Chicago, Chicago, IL, USA

Correspondence: Dr JR Turner, MD, PhD, Department of Pathology, University of Chicago, 5841 South Maryland Avenue, MC 1089, Chicago, IL 60637, USA. E-mail: jturner@bsd.uchicago.edu

${ }^{3}$ These authors contributed equally to this work.

Received 21 June 2008; revised 11 July 2008; accepted 14 July 2008
} 
invasion, and increased patient survival. ${ }^{25}$ Although the mechanism whereby claudins participate in tumorigenesis is poorly understood, it is interesting to note that $\beta$-catenin and claudin- 1 colocalize in the nucleus of many metastatic colonic adenocarcinomas, ${ }^{28}$ and genetic overexpression of claudin-1 increases metastatic potential of tumor cell lines. ${ }^{13}$ Conversely, inhibition of claudin-1 expression reduces $\beta$-catenin/Tcf/lef signaling in vitro. ${ }^{13}$ Together with claudin-2 promoter activation by $\beta$-catenin, ${ }^{29}$ these data suggest that the interplay between claudins and the APC/ $\beta$-catenin pathway may contribute to colonic neoplasia.

Although claudin expression and distribution has been studied extensively in IBD, ${ }^{3,4,8}$ the relationship between claudin modifications and IBD-associated neoplasia has not been examined. However, it is well established that patients with IBD are at increased risk of developing colorectal carcinoma. ${ }^{30-32}$ Emerging data suggest that the risk of cancer in IBD may be related to the severity of inflammation, ${ }^{33,34}$ suggesting that chronic injury may accelerate or otherwise contribute to neoplastic transformation. To define molecular mechanisms underlying this process, we asked if the altered expression and distribution of claudin proteins described in IBD was related to IBD-associated neoplasia. To test this we evaluated the expression of claudin-1, claudin-2, and claudin-4 in human biopsies of patients with IBD and IBD-associated dysplasia as well as in sporadic nonIBD-associated adenomas. Claudin- 1 and claudin- 2 were elevated in sporadic adenomas, IBD, and IBD-associated dysplasia. Nuclear localization of $\beta$-catenin, a correlate of $\beta$-catenin activation, was increased in all conditions where claudin-1 and claudin-2 upregulation occurred. In contrast, expression of claudin- 1 and claudin- 2 and nuclear localization of $\beta$-catenin were only minimally affected in acute, self-limited colitis (ASLC). These data suggest that claudin-1 and claudin-2 expression may be regulated by $\beta$-catenin in chronic, but not acute, inflammatory conditions. Furthermore, the data indicate a potential association between increased claudin-1 and claudin- 2 expression and elevated neoplastic risk in IBD.

\section{MATERIALS AND METHODS Patient Material}

All patient materials for this study were obtained under a protocol approved by The University of Chicago Institutional Review Board. Formalin-fixed, paraffin-embedded tissue from biopsy and colectomy specimen included 16 specimens from 15 IBD patients with colonic epithelial dysplasia (12 ulcerative colitis (UC) and 3 Crohn's colitis). Tissues from 12 patients were selected from biopsies deemed to have ASLC based on the presence of normal crypt architecture and acute inflammation and the absence of abnormal crypt architecture, crypt atrophy, mixed lamina propria inflammation, basal plasmacytosis, basal lymphoid aggregates, and basal lymphoid hyperplasia. ${ }^{35-38}$ All cases of ASLC were reviewed by at least three GI pathologists and, for a case to be included, all three had to agree that there were no features of IBD. In addition, no ASLC patients had history of IBD or developed biopsy demonstrated in IBD in 1- to 2-year interval since the biopsy. Sporadic adenomas and invasive carcinomas were from patients without IBD who underwent colectomy for adenocarcinoma (36 patients). Control specimens were collected from the margins of these colectomies.

\section{Tissue Microarray and Sectioning}

Tissue microarrays containing 36 sporadic adenomas, normal adjacent colonic mucosa from each adenoma, and 3 samples of invasive carcinoma were prepared from paraffin tissue blocks. For each area, a minimum of two tissue cylinders with a diameter of $1.5 \mathrm{~mm}$ were arrayed into a recipient block with a manual tissue microarrayer (Beecher Instruments, Sun Prairie, WI, USA). The recipient tissue microarray block was cut into $4 \mu \mathrm{m}$ thick sections for analysis by immunohistochemistry. After sectioning the array block, between 32 and 36 cores were available for staining on each slide. For the remaining 28 cases of ASLC and IBD that were not included in the array, tissue was immunostained using traditional paraffin sections.

\section{Immunohistochemical Staining}

Immunostaining was performed on $4 \mu \mathrm{m}$ thick, formalin fixed, paraffin-embedded tissue sections mounted on positively charged X-tra slides (Surgipath, Richmond, IL, USA). Paraffin sections were deparaffinized in xylene, rehydrated, and washed in Tris-buffered saline. Endogenous peroxidase activity was quenched by incubation in $3 \% \mathrm{H}_{2} \mathrm{O}_{2}$ in methanol for $5 \mathrm{~min}$. Nonspecific binding sites were blocked using nonserum Protein Block (DAKO, Carpinteria, CA, USA) for $20 \mathrm{~min}$. Antigen retrieval for monoclonal anticlaudin-2 (clone 12H12; Invitrogen, Carlsbad, CA, USA), anti-claudin-4 (clone 3E2C1; Invitrogen), and monoclonal anti- $\beta$-catenin (clone 14; BD Biosciences, San Jose, CA, USA) staining was carried out by heating sections in citrate buffer $(\mathrm{pH}=6)$ for $15 \mathrm{~min}$ in a microwave oven. Sections to be stained with polyclonal anti-claudin-1 (JAY.8; Invitrogen) were heat antigen-retrieved in EDTA buffer $(\mathrm{pH}=9)$. For polyclonal rabbit anti-occludin (catalog number 71-1500; Invitrogen) staining, antigen was retrieved by combined enzymatic digestion with $0.04 \%$ protease solution in distilled water followed by heat retrieval in citrate buffer $(\mathrm{pH}=6)$ for $15 \mathrm{~min}$ in a microwave oven. Tissue sections were incubated for $1 \mathrm{~h}$ at room temperature in a humidity chamber with primary antibodies diluted to $2.5 \mu \mathrm{g} / \mathrm{ml}$ (claudin- 1 and occludin), $5 \mu \mathrm{g} / \mathrm{ml}$ (claudin-2 and claudin-4), and $1.25 \mu \mathrm{g} / \mathrm{ml}$ ( $\beta$-catenin) using antibody diluent buffer (DAKO). After three washes, slides were incubated for $30 \mathrm{~min}$ with goat antirabbit or anti-mouse immunoglobulin conjugated to a horseradish peroxidase-labeled polymer (EnvisionTM+ System; DAKO). After washing, slides were developed for 
5 min with $3,3^{\prime}$-diaminobenzidine chromogen and counterstained with hematoxylin.

\section{Analysis of Immunohistochemical Staining and Inflammatory Activity}

Staining intensity of all immunostains was scored semiquantitatively from 0 to 3 by two separate observers. When scores between the two observers were discordant, a consensus was achieved by conference at a two-headed microscope. Hematoxylin and eosin-stained slides were graded for inflammation from 0 to 3 using the criteria shown in Table 1.

Student's $t$-test was used to determine differences in expression between tissue groups. Pearson correlation was used to determine correlations between degrees of active inflammation and expression. For $t$-tests and Pearson's correlation, statistical significance was taken as $P \leq 0.05$.

\section{RESULTS}

\section{Patient Population}

The patient population for this study included 15 patients with IBD and dysplasia, 12 patients with ASLC, 36 patients with sporadic adenomas, and 3 cases of invasive colonic adenocarcinoma. Medications, at the time of biopsy or resection, were diverse within this patient population and precluded meaningful statistical analysis. Specimens were distributed throughout the right and left colon and there was no apparent association between location and tight junction protein expression. Patient information is summarized in Table 2. Inflammatory activity has been associated with risk of neoplasia in UC, and we have previously noted that expression of myosin light chain kinase, a regulator of tight junction permeability in colitis, ${ }^{39}$ correlates with histologic inflammatory activity. ${ }^{40}$ Thus, the inflammatory activity of all specimens was graded semiquantitatively (Table 3 ). All control tissues were free of active inflammation (grade 0 ). Sporadic adenomas often had increased intraepithelial or lamina propria granulocytes. In the three cases of carcinoma included in the tissue microarray, active inflammation was severe (grade 3). Intraepithelial granulocytes, with or without erosions, were the norm in ASLC.

\section{Table 1 Method of grading active inflammation}

\begin{tabular}{ll}
\hline Activity grade & Features \\
\hline Grade 0 (normal) & No features of acute or chronic injury \\
Grade 1 (inactive) & Architectural distortion, increased \\
& lamina propria lymphs, no activity \\
Grade 2 (active) & Increased lamina propria granulocytes \\
& with or without intraepithelial \\
Grade 3 (active) & granulocytes but without crypt abscesses \\
& Crypt abscesses or erosions \\
\hline
\end{tabular}

\section{Claudin-1 and Claudin-2 Expression is Increased in Active IBD}

Expression of claudin-1, claudin-2, claudin-4, and occludin was assessed in control tissues and those involved by IBD. Claudin-1 did not localize specifically to the region of the tight junction, but was present along the lateral membrane and also detected weakly within cytoplasmic granules of crypt colonocytes in control tissue (Figure 1). Expression was significantly increased within epithelia adjacent to lymphoid aggregates, where claudin-1 was localized to cytoplasmic granules and colonocyte lateral membranes (Figure 1; $P<0.05)$.

Claudin-1 expression in inactive IBD was similar to that in normal colon. In contrast, expression of claudin- 1 was significantly increased when inflammatory activity was present $(P<0.001)$. These increases in claudin-1 expression were progressive, with greater expression correlated with inflammatory activity grade in IBD $(r=0.75, P<0.001)$. In addition, active inflammation was associated with greater localization of claudin-1 to lateral membranes (Figure 1). Claudin-1 expression was similar in colonic mucosa from UC and Crohn's disease (CD) patients.

In contrast to claudin-1, claudin-2 expression in surface colonocytes was most intense in the apical cytoplasm and tight junction region. Claudin-2 expression in crypt epithelium was punctate and confined to lateral cell membranes and cytoplasm (Figure 1). There was no change in claudin-2 expression or localization in mucosa near lymphoid aggregates and expression in inactive IBD was similar to control tissue (Figure 1). Like claudin 1, claudin-2 was increased in IBD when active inflammation was present $(P<0.05)$, but was not increased in IBD with grade 1 inflammatory activity. Claudin-2 expression correlated positively with claudin-1 expression $(r=0.626, P<0.001)$ and disease activity $(r=0.451, P<0.01)$. Increased claudin-2 expression was noted in lateral membranes, cytoplasmic granules, and diffusely within the cytoplasm (Figure 1). Claudin-2 expression was similar in cases of UC and CD.

Claudin-4 was detected primarily in lateral membranes of surface and crypt colonocytes (Figure 1). In some cases of inactive IBD there was an increase in lateral membrane accumulation of claudin- 4 that did not reach statistical significance (Figure $1 ; P=0.15$ ). Claudin-4 expression was enhanced in the presence of grade 2 inflammatory activity, where intense membranous staining was present $(P<0.05 v s$ control). However, claudin-4 expression was variable, and not significantly increased compared to control, in IBD with grade 3 inflammatory activity. There was no difference in claudin-4 expression when UC and CD cases were compared.

Of the tight junction proteins studied, occludin demonstrated the greatest localization to the region of the tight junction. However, weak cytoplasmic staining was also present. Occludin expression and localization in both inactive and active IBD were similar to that in control tissue (Figure 1). 
Table 2 Patient demographics

\begin{tabular}{|c|c|c|c|c|c|}
\hline & Sporadic adenoma $^{\mathrm{a}}$ & UC & $C D$ & Total IBD & ASLC \\
\hline Number of patients & 36 & 12 & 3 & 15 & 12 \\
\hline Female:male & $14: 22$ & $5: 7$ & $2: 1$ & $7: 8$ & $6: 6$ \\
\hline Family history IBD, \% $\left(n^{\mathrm{b}}\right)$ & & $56(9)$ & $100(1)$ & $60(10)$ & \\
\hline Smoking history, \% $\left(n^{\mathrm{b}}\right)$ & & $27(11)$ & $0(2)$ & $23(13)$ & \\
\hline \multicolumn{6}{|l|}{ Location } \\
\hline Right colon & $67 \%(24)$ & $33 \%(4)$ & $67 \%(2)$ & $40 \%(6)$ & $42 \%(5)$ \\
\hline Left colon & $33 \%(12)$ & $67 \%(8)$ & $33 \%(1)$ & $60 \%(9)$ & $58 \%(7)$ \\
\hline Sulfasalazine & & 2 & 0 & 2 & \\
\hline Belladonna alkaloids & & 1 & 0 & 1 & \\
\hline Mesalamine & & 0 & 1 & 1 & \\
\hline 6-Mercaptopurine & & 1 & 0 & 1 & \\
\hline Balsalazide disodium & & 1 & 0 & 1 & \\
\hline
\end{tabular}

Abbreviations: ASLC: acute, self-limited colitis; CD: Crohn's disease; IBD: inflammatory bowel disease; UC: ulcerative colitis.

${ }^{a}$ Control tissues were also from these patients.

${ }^{b}$ Number for whom data was available.

Table 3 Inflammatory grades of samples analyzed

\begin{tabular}{|c|c|c|c|c|}
\hline & Grade 0 & Grade 1 & Grade 2 & Grade 3 \\
\hline Control & 36 & & & \\
\hline IBD & & 4 & 7 & 5 \\
\hline ASLC & & & 8 & 4 \\
\hline
\end{tabular}

Abbreviations: ASLC: acute, self-limited colitis; IBD: inflammatory bowel disease.

\section{Claudin-1 and Claudin-2 Expression is not Increased in ASLC}

Expression of claudin-1 and claudin-2 is increased in active IBD, but not inactive disease. Thus, increased expression of the claudin-1 and claudin-2 may be related to IBD or, alternatively, merely to the presence of active inflammation. To discriminate between these possibilities, we studied claudin expression in cases of ASLC. Claudin-1 expression was similar to control, noninflamed tissue in 9 of 12 cases of ASLC cases and was scored as 2 or greater in only 3 cases. This was only slightly greater than claudin-1 expression in control tissues (Figure 2), where 1 of 33 cases received a score of 2 and none were scored as $3(P<0.05)$. Importantly, claudin-1 expression in ASLC was significantly lower than in cases of active IBD, in which 9 of 12 cases were scored 2 or greater $(P<0.005)$. Expression of claudin-2 in ASLC was limited and was not significantly different from control (Figure 2). Claudin-4 expression in ASLC with grade 2 inflammation was increased to an extent similar to that observed in IBD with grade 2 inflammatory activity (Figure 2; $P<0.001$ ). Taken together, it appears that the overall expression of claudin-1 and claudin-2 is differently regulated in ASLC relative to IBD. In contrast, the data suggest that the increases in claudin- 4 expression observed in IBD may be related to active inflammation. Although claudin-1 and claudin-2 expression is modified by active inflammation, this regulation in IBD is distinct from the regulation in ASLC.

\section{Expression of Claudin-1 and Claudin-2 is Increased in Sporadic Adenomas}

Although increased tight junction protein expression has been assessed in colorectal adenocarcinoma, there are no reports examining expression in preinvasive dysplastic lesions. We therefore studied expression of claudin-1, claudin-2, claudin-4, and occludin in sporadic adenomas. As in invasive cancers, claudin- 1 and claudin- 2 expression was increased in adenomas (Figure 3; $P<0.001$ ). Claudin-1 was 

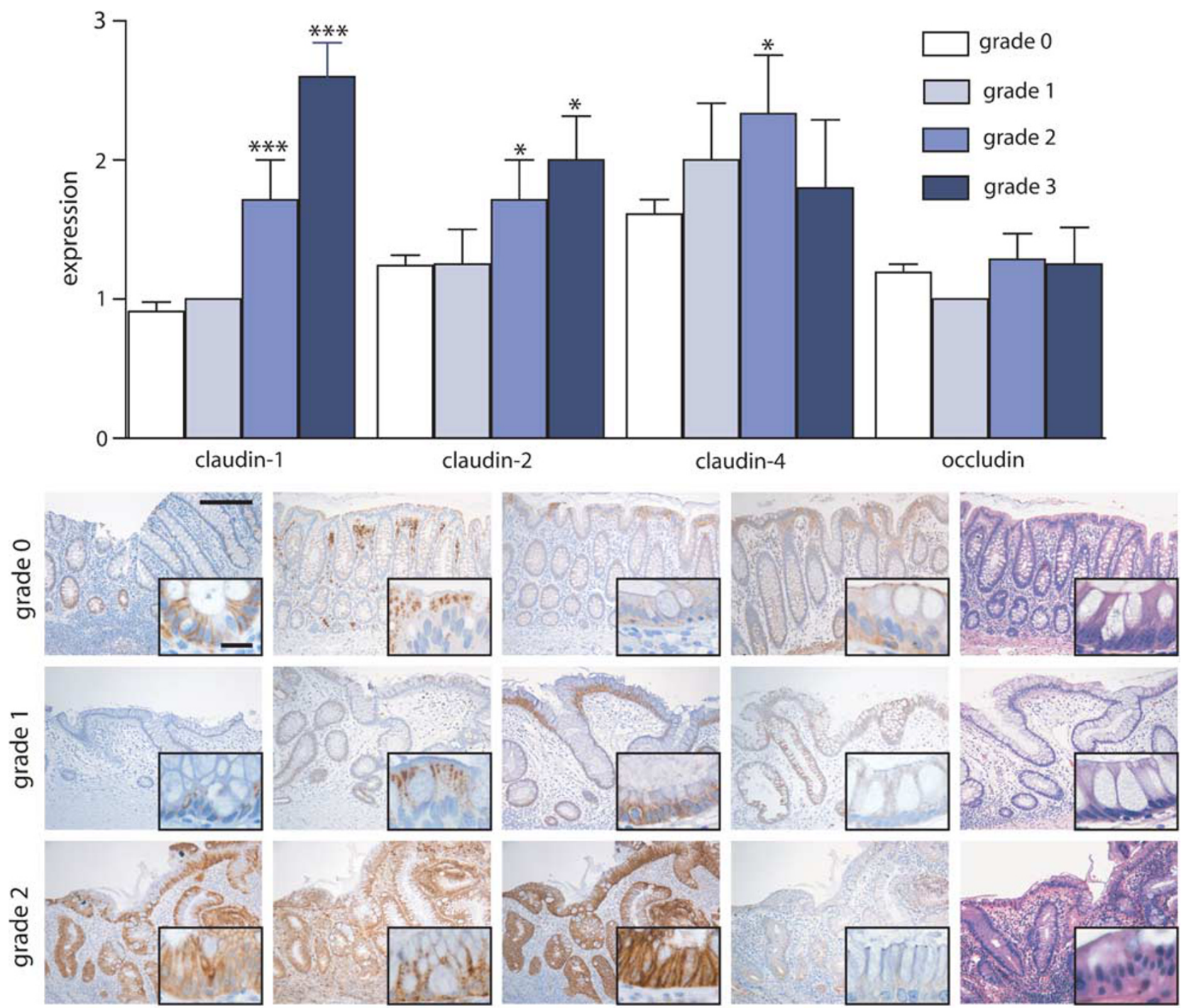

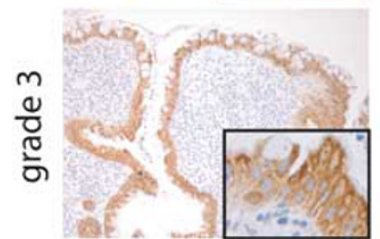

claudin-1

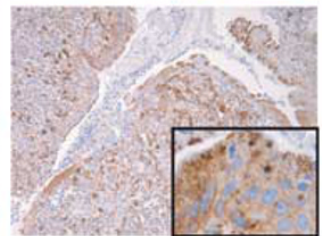

claudin-2

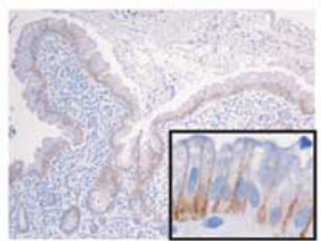

claudin-4

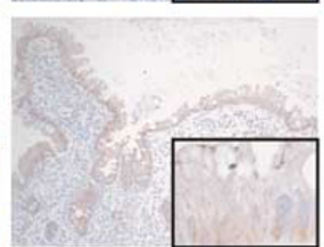

occludin

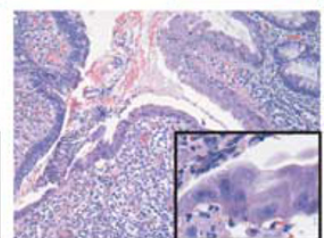

Figure 1 Claudin expression is elevated in IBD. Immunohistochemical staining in control and IBD was scored semiquantitatively from 0 to 3 and compared to grade of inflammation. Inflammatory grades 1-3 are from patients with IBD. Control tissue (grade 0 ) is from the normal margins of patients without IBD who underwent resection for large adenoma or carcinoma. Expression of claudin-1 and claudin-2 is increased in an inflammation-dependent manner, whereas claudin-4 expression is more variably increased. Occludin expression was constant in all conditions. Comparison of expression in IBD to control tissue: ${ }^{\star} P \leq 0.05 ;{ }^{* *} P \leq 0.001$. Scale bar $=200 \mu \mathrm{m}$, inset scale bar $=20 \mu \mathrm{m}$.

localized to lateral membranes of dysplastic colonocytes whereas claudin-2 was concentrated within apical cytoplasmic granules in dysplastic colonocytes. However, increased claudin- 1 and claudin-2 expression, which was seen in over $90 \%$ of sporadic adenomas, was the most remarkable change. We also evaluated staining of three representative cases of invasive carcinoma and observed similar strong positive staining (claudin- $1=2.00 \pm 0.00$, claudin- $2=2.33 \pm 0.33)$. Claudin- 4 and occludin expression and distribution were not significantly different between control, adenoma, or invasive carcinoma tissue.

\section{Claudin-1 and Claudin-2 Expression is Increased in IBD-Associated Dysplasia}

Although IBD confers an increased risk of colorectal carcinoma, the mechanisms of neoplasia in IBD patients may 

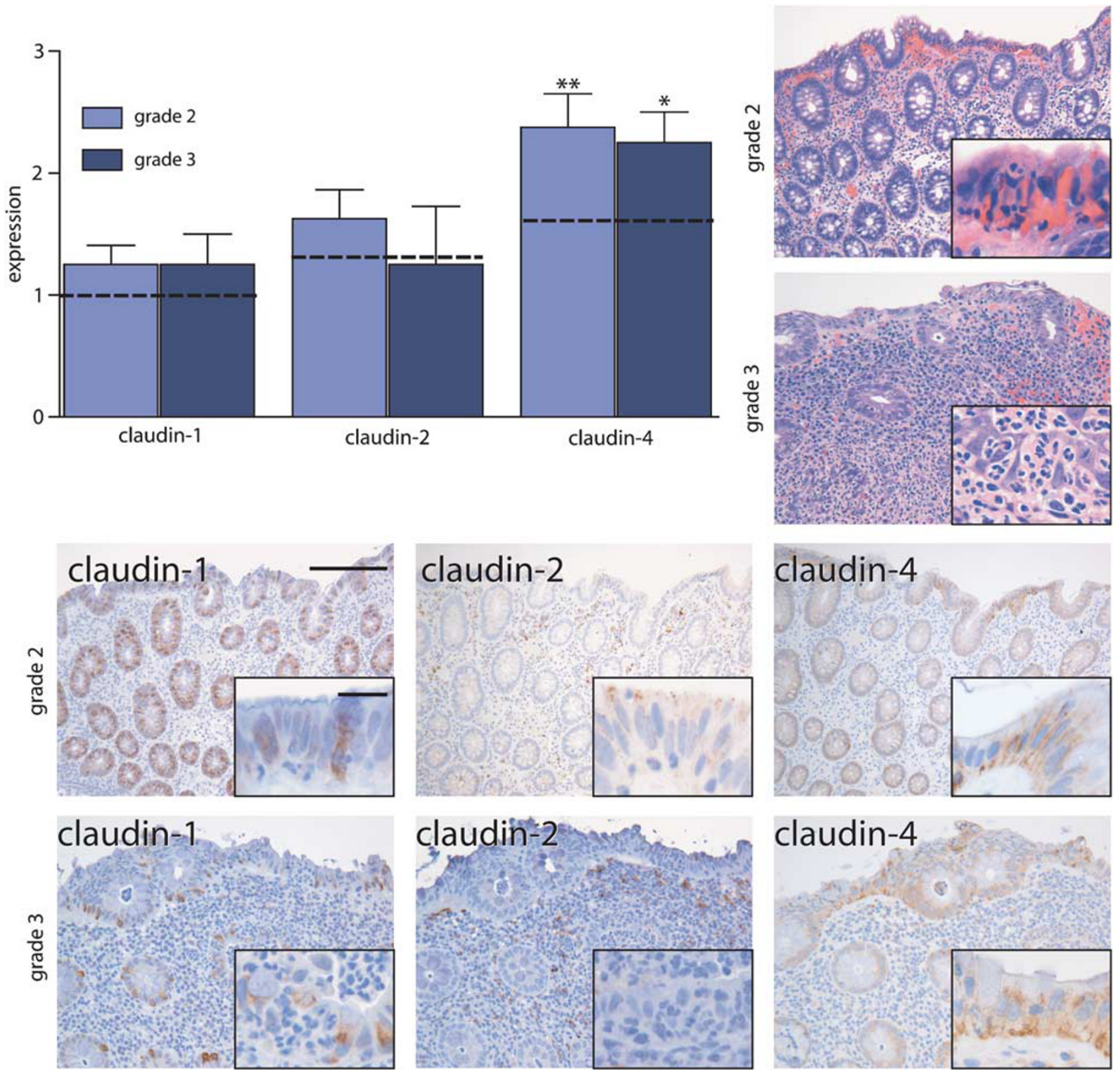

Figure 2 Claudin-1 and claudin-2 expression is not elevated in ASLC. Immunohistochemical staining in grade 2 and grade 3 ASLC was scored semiquantitatively from $0-3$. Expression in control tissue (grade 0 ) is indicated by dashed lines. In contrast to the observations in IBD specimens, claudin-1 and claudin-2 expression is not significantly increased in ASLC. ${ }^{*} P \leq 0.05 ;{ }^{*} P \leq 0.01$. Scale bar $=200 \mu \mathrm{m}$, inset scale bar $=20 \mu \mathrm{m}$.

differ from those in patients with sporadic cancer. We therefore asked, given the increased expression of claudin-1 and claudin-2 in both IBD and sporadic adenomas, whether the same alterations would be present in IBD-associated dysplasia. Claudin-1 and claudin-2 expression in IBD-associated dysplasia was increased relative to nondysplastic IBD $(P<0.005)$ and was similar to that of sporadic adenomas (Figure 4). In contrast, claudin-4 and occludin were only slightly increased and not significantly different from nondysplastic IBD tissue. There were no differences between low and high-grade dysplasia for any of these tight junction proteins. There were also no difference between UC- and $\mathrm{CD}$-associated dysplasia.

\section{Nuclear $\boldsymbol{\beta}$-Catenin Localization is Increased in IBD}

Claudin-1 and claudin-2 expression has been reported to be modulated by $\beta$-catenin/Tcf/lef signaling and, conversely, claudin- 1 may regulate $\beta$-catenin. ${ }^{28}$ We therefore asked if the observed increases in claudin- 1 and claudin- 2 expression in IBD, IBD-associated dysplasia, and sporadic adenomas are associated with increased $\beta$-catenin nuclear localization, a marker of $\beta$-catenin activation. $\beta$-Catenin showed weak 

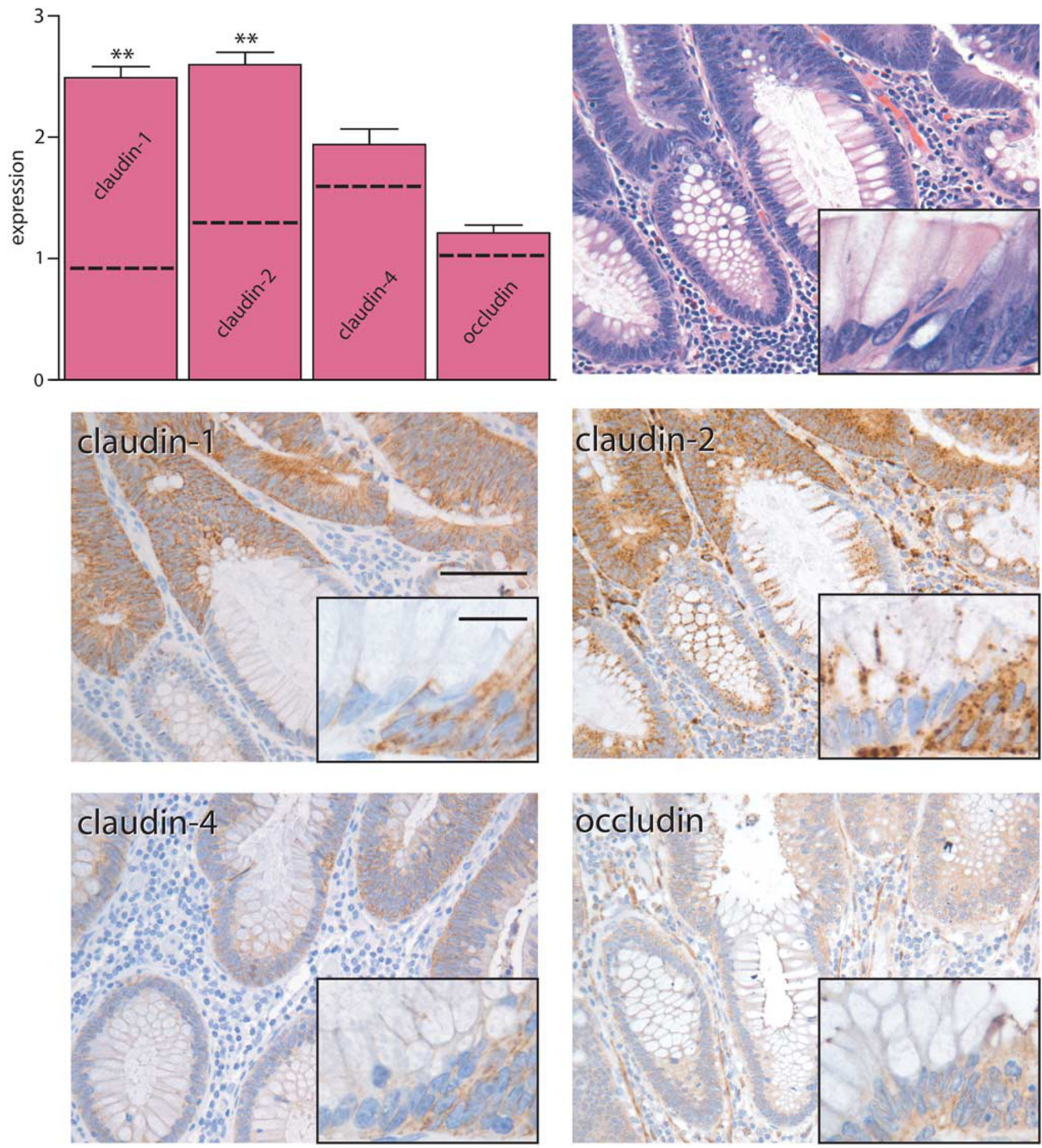

Figure 3 Claudin-1 and claudin-2 expression is elevated in sporadic adenomas. Immunohistochemical staining in sporadic adenomas was scored semiquantitatively from $0-3$. Claudin-1 and claudin-2 expression is significantly increased in dysplasia relative to nondysplastic tissue (dashed lines). ${ }^{*} P \leq 0.01$. Scale bar $=80 \mu \mathrm{m}$, inset scale bar $=20 \mu \mathrm{m}$.

nuclear localization in control tissues, most prominently in crypts. This was increased in sporadic adenomas (Figure 5a; $P<0.001)$. Nuclear $\beta$-catenin localization was also elevated in IBD compared to control tissue $(P<0.001) ; 11$ of 16 IBD specimens had increased nuclear $\beta$-catenin ( 9 of $13 \mathrm{UC}$ and 3 of $3 \mathrm{CD}$ ). Overall, there was no correlation between nuclear $\beta$-catenin localization and disease activity in IBD $(r=-0.12$, $P>0.6)$, but nuclear localization did increase from grade 1 to grade 2 IBD $(P<0.05)$. Nuclear $\beta$-catenin in IBD-associated dysplasia was greater than that in nondysplastic IBD 

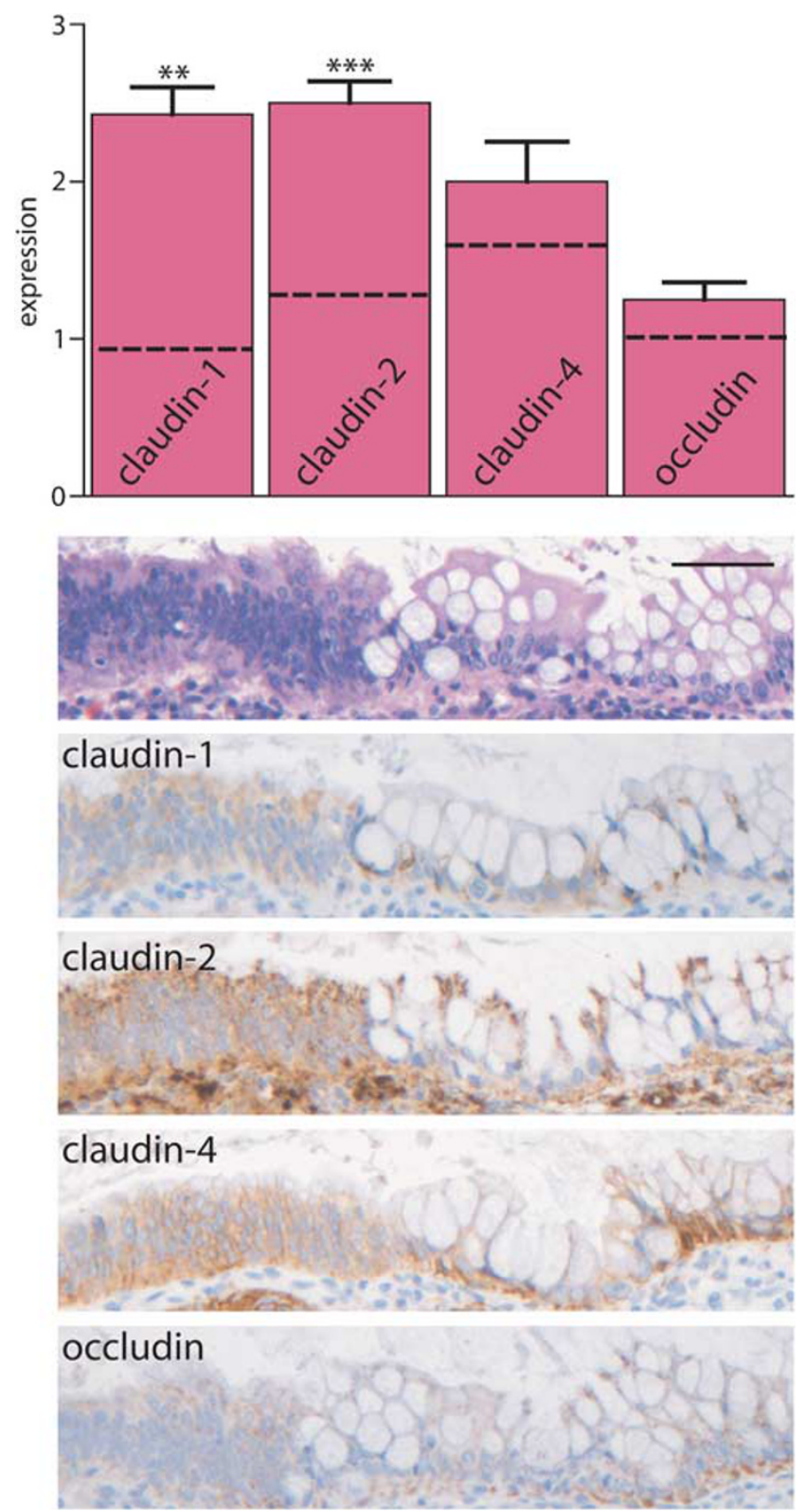

Figure 4 Claudin-1 and claudin-2 expression is elevated in IBD-associated dysplasia. Immunohistochemical staining in IBD dysplasia was scored semiquantitatively from 0 to 3 . Claudin- 1 and claudin- 2 expression is increased relative to control tissues (dashed lines). ${ }^{* * P} \leq 0.01 ;{ }^{* *} P \leq 0.001$. Scale bar $=60 \mu \mathrm{m}$.

$(P<0.005)$, and was quantitatively similar to staining in sporadic adenomas. In contrast, nuclear $\beta$-catenin was only increased in 3 of 12 cases of ASLC and did not correlate with disease activity (Figure 5). Thus, like claudin-1 and claudin-2, $\beta$-catenin staining is increased in IBD and dysplasia (sporadic and IBD associated), but is not increased in most cases of ASLC. Thus, activation of $\beta$-catenin may explain increases in claudin- 1 and claudin- 2 expression in IBD and IBD-associated dysplasia.

\section{DISCUSSION}

Patients with IBD have an increased propensity to develop colon carcinoma. ${ }^{30-34,41}$ The mechanisms underlying this susceptibility are not well understood, and the molecular events that contribute to this phenotype are ill defined. On the basis of previous data suggesting that expression of claudin family members is altered in IBD and invasive colon cancer, ${ }^{13,15-23,25-27}$ we hypothesized that modified expression of tight junction proteins could be associated with the increased risk of colon carcinoma in IBD. However, claudin family member distribution and expression has not been characterized in either sporadic or colitis-associated premalignant colonic dysplasia.

Claudin-1 protein has been reported to be slightly decreased in IBD on the basis of SDS-PAGE and immunoblot analysis of whole mucosal homogenates. ${ }^{4,8}$ However, claudin-1 expression and localization within colonocytes of IBD patients have not been assessed in the context of inflammation grade. We observed increased claudin-1 expression in colonocytes of IBD patients. This was linked to active inflammation, as claudin-1 expression was not increased in inactive IBD. Although the number of $\mathrm{CD}$ cases with dysplasia was small, it is interesting to note that overall results were similar in UC and CD. In agreement with previous immunohistochemical and SDS-PAGE immunoblot analyses, ${ }^{3,4,8}$ we found that claudin-2 expression is increased in colonocytes of patients with active IBD. This is consistent with the reported in vitro effects of IL-13 and TNF on claudin-2 expression. ${ }^{3,8}$ As these cytokines are also elevated in active $\mathrm{UC}^{8,42}$ and $\mathrm{CD},{ }^{9}$ respectively, they may explain the similar elevations of claudin-2 expression in these diseases. Importantly, the mere presence of active inflammation was not sufficient to increase expression of either claudin-1 or claudin-2, as expression was unaffected in ASLC. This also implies that increases in mitotic activity alone cannot explain our results.

In contrast to claudin-1 and claudin-2, claudin-4 expression was not correlated with disease activity. The observation is consistent with a previous study that used the same antibody used here to detect claudin $-4 .^{3}$ Moreover, as claudin-4 expression was increased in ASLC, the data suggest that the observed effects may be simply due to inflammatory activity and not a consequence of the chronic disease process.

To assess the association between altered claudin expression in IBD and neoplastic risk, we compared expression in nonneoplastic IBD to that in IBD-associated dysplasia and sporadic adenomas. Similar increases in expression of both claudin-1 and claudin-2 were present in IBD-associated dysplasia and sporadic adenomas. Given the emerging association of neoplastic risk in IBD with inflammatory activity, ${ }^{33,34}$ these data suggest that increased expression of claudin-1 and claudin-2 in this setting may contribute to neoplastic progression. Although these changes in claudin-1 and claudin-2 expression are expected to impact barrier 


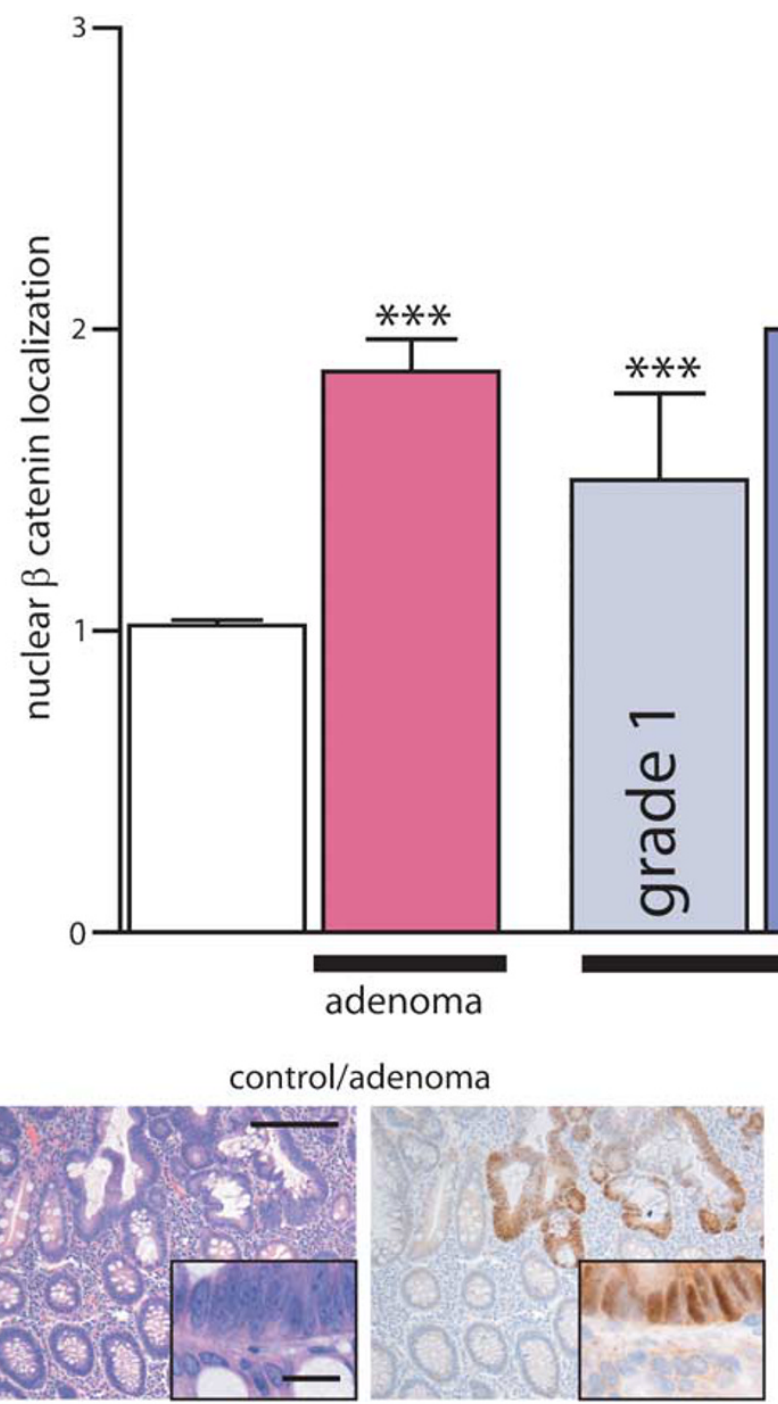

grade 2 IBD

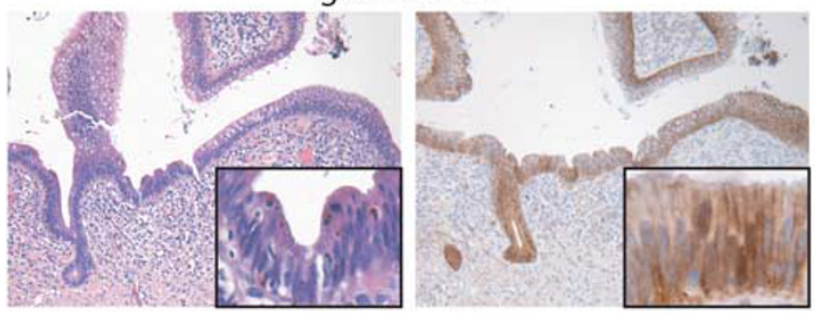

grade 2 ASLC
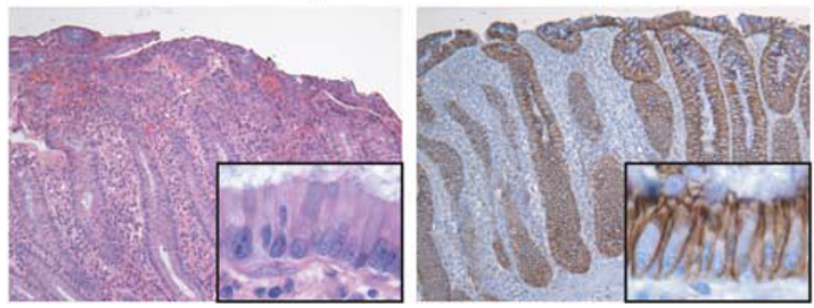

$* * *$

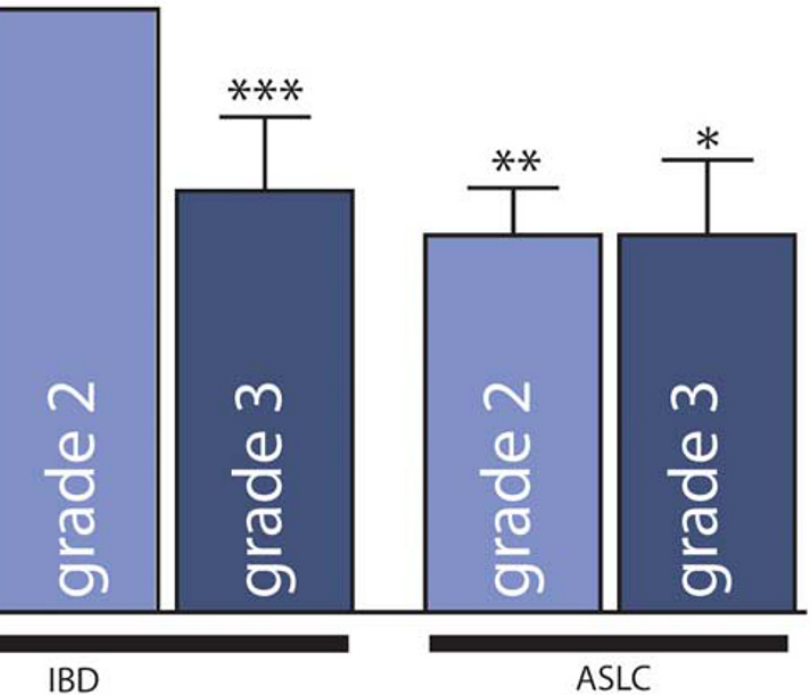

grade 1 IBD

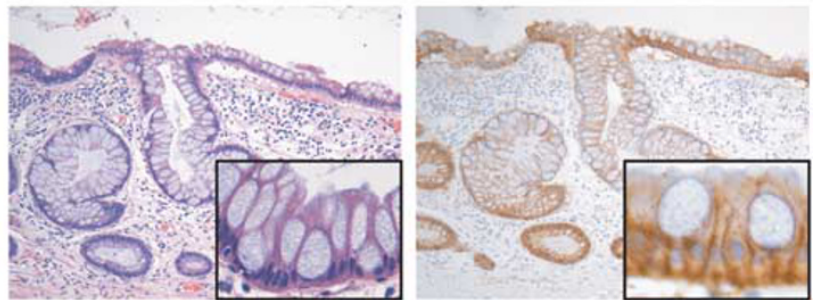

grade 3 IBD

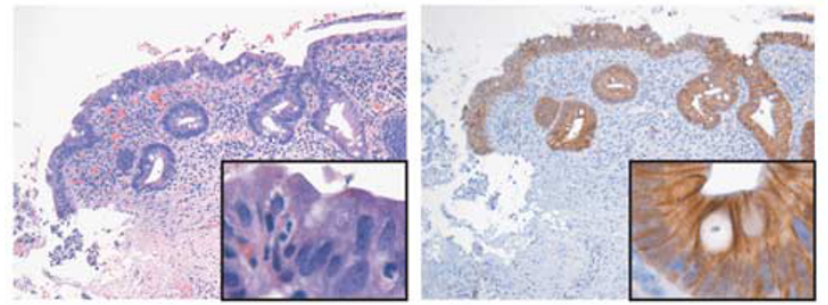

grade 3 ASLC
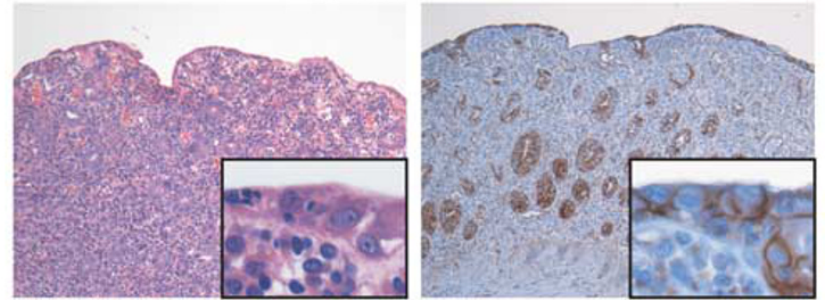

Figure 5 Nuclear $\beta$-catenin localization is enhanced in dysplasia and IBD. Nuclear $\beta$-catenin localization was assessed semiquantitatively and compared to control tissue (white bar). $\beta$-Catenin within the nucleus was increased in sporadic adenomas, IBD, and, to a lesser extent in ASLC. ${ }^{\star P} \leq 0.05$; ${ }^{*} P \leq 0.01$; ${ }^{* *} P \leq 0.001$. Scale bar $=200 \mu \mathrm{m}$, inset scale bar $=20 \mu \mathrm{m}$. 
function, it is not clear that barrier modulation is the mechanism by which claudin protein expression influences neoplasia. It is possible that claudin proteins interact with signaling pathways, including TGF $\beta / \mathrm{SMAD}$ and $\beta$-catenin, ${ }^{13,43}$ in a manner that is separate from their effects on barrier function.

Although the relationship we have identified between claudin-1 and claudin-2 expression and active inflammation in IBD is correlative, the previous observation that these tight junction proteins can either regulate or be regulated by $\beta$-catenin/Tcf/lef signaling ${ }^{13,28,29}$ suggests that the increased claudin-1 and claudin-2 expression observed may be due to $\beta$-catenin activation. We therefore assessed $\beta$-catenin activation. As expected, increased translocation of $\beta$-catenin from the lateral membranes to the nucleus was present in sporadic adenomas. Strikingly, this enhanced nuclear localization was also present in IBD and IBD-associated dysplasia. These observations, together with previous in vitro analyses, point to $\beta$-catenin as an upstream regulator of claudin- 1 and claudin-2 in IBD.

Some discrepancies exist among studies of claudin family member expression in neoplasia, but this may, in part, be due to differences in tumor stage. For example, although we observed increases in claudin- 1 and claudin- 2 expression in adenomas and IBD-associated dysplasia, others have reported reduced claudin expression in high-grade colorectal carcinomas. ${ }^{25}$ This may reflect the loss of proteins associated with epithelial differentiation during epithelial to mesenchymal transformation. Therefore, although claudin-1 and claudin-2 are elevated in preinvasive IBD-associated and sporadic neoplasia, expression may not remain increased in invasive lesions.

In summary, these data suggest that active inflammation in the setting of chronic IBD results in an increase in $\beta$-catenin transcriptional activity that may contribute to increased claudin-1 and claudin-2 expression. The data support the hypothesis that elevated claudin-1 and claudin-2 expression contribute to neoplastic transformation and provide human data that corroborate previous work using cell lines and tumor xenografts. ${ }^{13}$ Thus, increased claudin-1 and claudin-2 expression may contribute to carcinogenesis in IBD.

\section{ACKNOWLEDGEMENTS}

We thank Can Gong and Leslie Martin for excellent technical assistance, Cami McBride for statistical consultation, and Alana Bunnag for chart review. This work was supported by The National Institutes of Health (Grants R01DK061931, R01DK068271, P30CA14599, T32HL007237, and F32DK082134).

1. Schmitz H, Barmeyer $\mathrm{C}$, Fromm M, et al. Altered tight junction structure contributes to the impaired epithelial barrier function in ulcerative colitis,. Gastroenterology 1999;116:301-309.

2. Clayburgh DR, Shen L, Turner JR. A porous defense: the leaky epithelial barrier in intestinal disease. Lab Invest 2004;84:282-291.

3. Prasad $\mathrm{S}$, Mingrino $\mathrm{R}$, Kaukinen $\mathrm{K}$, et al. Inflammatory processes have differential effects on claudins 2, 3 and 4 in colonic epithelial cells. Lab Invest 2005;85:1139-1162.
4. Zeissig S, Burgel N, Gunzel D, et al. Changes in expression and distribution of claudin 2, 5 and 8 lead to discontinuous tight junctions and barrier dysfunction in active Crohn's disease. Gut 2007;56:61-72.

5. Weber CR, Turner JR. Inflammatory bowel disease: is it really just another break in the wall? Gut 2007;56:6-8.

6. Clayburgh DR, Barrett TA, Tang Y, et al. Epithelial myosin light chain kinase-dependent barrier dysfunction mediates $T$ cell activationinduced diarrhea in vivo. J Clin Invest 2005;115:2702-2715.

7. Graham WV, Wang F, Clayburgh DR, et al. Tumor necrosis factorinduced long myosin light chain kinase transcription is regulated by differentiation-dependent signaling events. Characterization of the human long myosin light chain kinase promoter. J Biol Chem 2006;281:26205-26215.

8. Heller F, Florian P, Bojarski C, et al. Interleukin-13 is the key effector Th2 cytokine in ulcerative colitis that affects epithelial tight junctions, apoptosis, and cell restitution. Gastroenterology 2005;129:550-564.

9. MacDonald $T$, Hutchings $P$, Choy $M-Y$, et al. Tumour necrosis factoralpha and interferon-gamma production measured at the single cell level in normal and inflamed human intestine. Clin Exp Immunol 1990;81:301-305.

10. Mankertz J, Tavalali S, Schmitz $\mathrm{H}$, et al. Expression from the human occludin promoter is affected by tumor necrosis factor alpha and interferon gamma. J Cell Sci 2000;113(Part 11):2085-2090.

11. Wang $F$, Graham WV, Wang $Y$, et al. Interferon-gamma and tumor necrosis factor-alpha synergize to induce intestinal epithelial barrier dysfunction by up-regulating myosin light chain kinase expression. Am J Pathol 2005;166:409-419.

12. Wang F, Schwarz BT, Graham WV, et al. IFN-gamma-induced TNFR2 expression is required for TNF-dependent intestinal epithelial barrier dysfunction. Gastroenterology 2006;131:1153-1163.

13. Dhawan $P$, Singh $A B$, Deane NG, et al. Claudin-1 regulates cellular transformation and metastatic behavior in colon cancer. J Clin Invest 2005;115:1765-1776.

14. Matter K, Balda MS. Signalling to and from tight junctions. Nat Rev Mol Cell Biol 2003;4:225-236.

15. Hewitt KJ, Agarwal R, Morin PJ. The claudin gene family: expression in normal and neoplastic tissues. BMC Cancer 2006;6:186.

16. Kominsky SL. Claudins: emerging targets for cancer therapy. Expert Rev Mol Med 2006;8:1-11.

17. Soini Y. Expression of claudins 1, 2, 3, 4, 5 and 7 in various types of tumours. Histopathology 2005;46:551-560.

18. Swisshelm K, Macek R, Kubbies M. Role of claudins in tumorigenesis. Adv Drug Deliv Rev 2005;57:919-928.

19. Morin PJ. Claudin proteins in human cancer: promising new targets for diagnosis and therapy. Cancer Res 2005;65:9603-9606.

20. Michl P, Barth C, Buchholz M, et al. Claudin-4 expression decreases invasiveness and metastatic potential of pancreatic cancer. Cancer Res 2003;63:6265-6271.

21. Gyorffy H, Holczbauer A, Nagy P, et al. Claudin expression in Barrett's esophagus and adenocarcinoma. Virchows Arch 2005;447:961-968.

22. Lee SK, Moon J, Park SW, et al. Loss of the tight junction protein claudin 4 correlates with histological growth-pattern and differentiation in advanced gastric adenocarcinoma. Oncol Rep 2005;13:193-199.

23. Grone J, Weber B, Staub E, et al. Differential expression of genes encoding tight junction proteins in colorectal cancer: frequent dysregulation of claudin-1, -8 and -12 . Int J Colorectal Dis 2007;22: 651-659.

24. Kinugasa $T$, Huo Q, Higashi $D$, et al. Selective up-regulation of claudin-1 and claudin-2 in colorectal cancer. Anticancer Res 2007;27:3729-3734.

25. Resnick MB, Konkin T, Routhier J, et al. Claudin-1 is a strong prognostic indicator in stage II colonic cancer: a tissue microarray study. Mod Pathol 2005;18:511-518.

26. Aung PP, Mitani Y, Sanada Y, et al. Differential expression of claudin-2 in normal human tissues and gastrointestinal carcinomas. Virchows Arch 2006;448:428-434.

27. Hahn-Stromberg V, Edvardsson $\mathrm{H}$, Bodin $\mathrm{L}$, et al. Disturbed expression of E-cadherin, beta-catenin and tight junction proteins in colon carcinoma is unrelated to growth pattern and genetic polymorphisms. APMIS 2008;116:253-262.

28. Miwa N, Furuse M, Tsukita S, et al. Involvement of claudin-1 in the beta-catenin/Tcf signaling pathway and its frequent upregulation in human colorectal cancers. Oncol Res 2001;12:469-476. 
29. Mankertz J, Hillenbrand B, Tavalali S, et al. Functional crosstalk between Wnt signaling and Cdx-related transcriptional activation in the regulation of the claudin- 2 promoter activity. Biochem Biophys Res Commun 2004;314:1001-1007.

30. Eaden J. Review article: colorectal carcinoma and inflammatory bowel disease. Aliment Pharmacol Ther 2004;20(Suppl 4): 24-30.

31. von Roon AC, Reese G, Teare J, et al. The risk of cancer in patients with Crohn's disease. Dis Colon Rectum 2007;50:839-855.

32. Bernstein $\mathrm{CN}$, Blanchard JF, Kliewer $\mathrm{E}$, et al. Cancer risk in patients with inflammatory bowel disease: a population-based study. Cancer 2001;91:854-862.

33. Itzkowitz SH, Yio X. Inflammation and cancer IV. Colorectal cancer in inflammatory bowel disease: the role of inflammation. Am J Physiol Gastrointest Liver Physiol 2004;287:G7-G17.

34. Rutter M, Saunders B, Wilkinson K, et al. Severity of inflammation is a risk factor for colorectal neoplasia in ulcerative colitis. Gastroenterology 2004;126:451-459.

35. Kumar NB, Nostrant TT, Appelman HD. The histopathologic spectrum of acute self-limited colitis (acute infectious-type colitis). Am J Surg Pathol 1982;6:523-529.
36. Surawicz CM. The role of rectal biopsy in infectious colitis. Am J Surg Pathol 1988;12(Suppl 1):82-88.

37. Surawicz CM, Belic L. Rectal biopsy helps to distinguish acute selflimited colitis from idiopathic inflammatory bowel disease. Gastroenterol 1984;86:104-113.

38. Surawicz CM, Haggitt RC, Husseman M, et al. Mucosal biopsy diagnosis of colitis: acute self-limited colitis and idiopathic inflammatory bowel disease. Gastroenterol 1994;107:755-763.

39. Turner JR. Molecular basis of epithelial barrier regulation: from basic mechanisms to clinical application. Am J Pathol 2006;169:1901-1909.

40. Blair SA, Kane SV, Clayburgh DR, et al. Epithelial myosin light chain kinase expression and activity are upregulated in inflammatory bowel disease. Lab Invest 2006;86:191-201.

41. Vagefi PA, Longo WE. Colorectal cancer in patients with inflammatory bowel disease. Clin Colorectal Cancer 2005;4:313-319.

42. Fuss IJ, Heller F, Boirivant M, et al. Nonclassical CD1d-restricted NK T cells that produce IL-13 characterize an atypical Th2 response in ulcerative colitis. J Clin Invest 2004;113:1490-1497.

43. Shiou SR, Singh AB, Moorthy $K$, et al. Smad4 regulates claudin-1 expression in a transforming growth factor-beta-independent manner in colon cancer cells. Cancer Res 2007;67:1571-1579. 